\title{
When Allergen Immunotherapy Perfectly Meets its Need: A Case Report
}

Elisa Boni ${ }^{1}$, Cristoforo Incorvaia ${ }^{2}$, Elena Makrì ${ }^{2}$, Donatella Preziosi ${ }^{3}$ and Marina Mauro ${ }^{3}$

${ }^{1}$ Allergy Unit, ASL Alessandria, Alessandria, Italy

${ }^{2}$ Cardiac/Pulmonary Rehabilitation, ASST Gaetano Pini/CTO, Milan, Italy

${ }^{3}$ Allergy Unit, Sant'Anna Hospital, ASST Lariana, Como, Italy

*Corresponding author: Cristoforo Incorvaia, Cardiac/Pulmonary Rehabilitation, ASST Gaetano Pini/CTO, Via Bignami, 20100 Milan, Italy, Fax: +390257003579; Tel: +390257993289; E-mail: cristoforo.incorvaia@gmail.com

Received date: May 31, 2017; Accepted date: June 05, 2017; Published date: June 12, 2017

Copyright: @ 2017 Boni E, et al. This is an open-access article distributed under the terms of the Creative Commons Attribution License, which permits unrestricted use, distribution, and reproduction in any medium, provided the original author and source are credited.

\section{Abstract}

Seasonal, pollen-induced allergic rhinitis can be managed by symptomatic drug treatment, but only allergen immunotherapy (AIT) is able to work on the causes of allergy. Usually, the effectiveness of AIT is assessed by clinical criteria, though the ideal outcome is to lose the allergic sensitization to the administered allergen(s).

Here we report the case of a patient who after three years of AIT using a grass pollen extract containing Phleum pratense, Dactilys glomerata, Anthoxanthum odoratum, Poa pratensis and Lolium perenne but not Cynodon dactylon, to which the patient was also sensitized, developed a negative response to allergy tests to the administered allergens.

After 3 years of SCIT, the patient was free of both nose and lung symptoms during the grass pollen season and had negative results to the pollens included in the extract, while $C$. dactylon showed a decrease in respect to basal value but not a negative result.

The findings from this case show that AIT in optimal circumstances is able to achieve a complete tolerance to the administered allergen demonstrated by the development of negative results to the grass pollens contained in the extract used for the treatment. This confirms the recent definition of AIT as a treatment fulfilling the requirements of precision medicine.
\end{abstract}

Keywords: Allergen immunotherapy; Grass pollen; Allergic rhinitis

\section{Introduction}

As shown by aerobiological and allergological studies, grass pollen is a major cause of respiratory allergy worldwide [1-5]. Taxonomically, grasses belong to the family of Poaceae (Figure 1), the temperate Pooideae subfamily contains the most allergenic species, that include Lolium perenne, Phleum pratense, Anthoxanthum odoratum, Dactylis glomerata, Holcus lanatus, Poa pratensis, and others. However, there are further subfamilies that include species capable to induce allergic symptoms, such as Chloridoideae (Cynodon dactylon) and Triticeae, the latter including Triticum aestivum (wheat), Secale cereale (rye) and Hordeum vulgare (barley), that are involved in baker's asthma [6] Recently, to the usual method of pollen counts in the air that produces the pollen calendars and thus provide useful indications to allergic patients on the concentration of pollen grains during spring, though it is unable to distinguish at microscopy reading the different grasses, techniques such as molecular allergy diagnostics [7] and phenology [8] were introduced. The first enables to assess the occurrence in each grass species of the various allergen molecules; some of them are species-specific and others have a high grade of structural homology and thus are cross-reactive among a number of grasses. The main grass pollen allergens are included in the group 1 and group 5 molecules. According to the Linnaean denomination, they are known as Phl p 1, Dac g 1, Ant o 1, Hol 1 1, etc, and Phl p 5, Dac g 5, Ant o 5, Hol 1 5, etc [9]. Instead, the cross-reacting molecules include profilins and polcalcins that due to their almost ubiquitous occurrence are knowns as "panallergens" [10]. As far as phenology is concerned, it is feasible to directly detect the pollen release for each individual grass. This allowed to observe that the various species of grasses release their pollen grains at different times during the pollen season and thus to assess their relative clinical importance [8]. Grass pollen allergy may be treated by drugs acting on allergic symptoms, such as oral or nasal topical antihistamines and topical corticosteroids, but if the treatment is aimed at working on the causes of the disease only allergen immunotherapy (AIT) can be considered, in its two forms of subcutaneous immunotherapy (SCIT) and sublingual immunotherapy (SLIT) [11-13]. The efficacy of both SLIT and SCIT on grass polleninduced respiratory allergy is demonstrated by meta-analysis [14]. The mechanisms of action of AIT include stimulation to produce allergenspecific IgG blocking antibodies and changes in the balance between the different T-cell phenotypes, particularly by the induction of regulatory T-cell subtypes that have been detected with both routes of administration [15]. These immunological modifications are mirrored by the development of progressive clinical tolerance to the administered allergens. On the other hand, in the natural history of allergy the levels of specific IgE, and consequently the clinical symptoms, may spontaneously decrease over time [16]. 


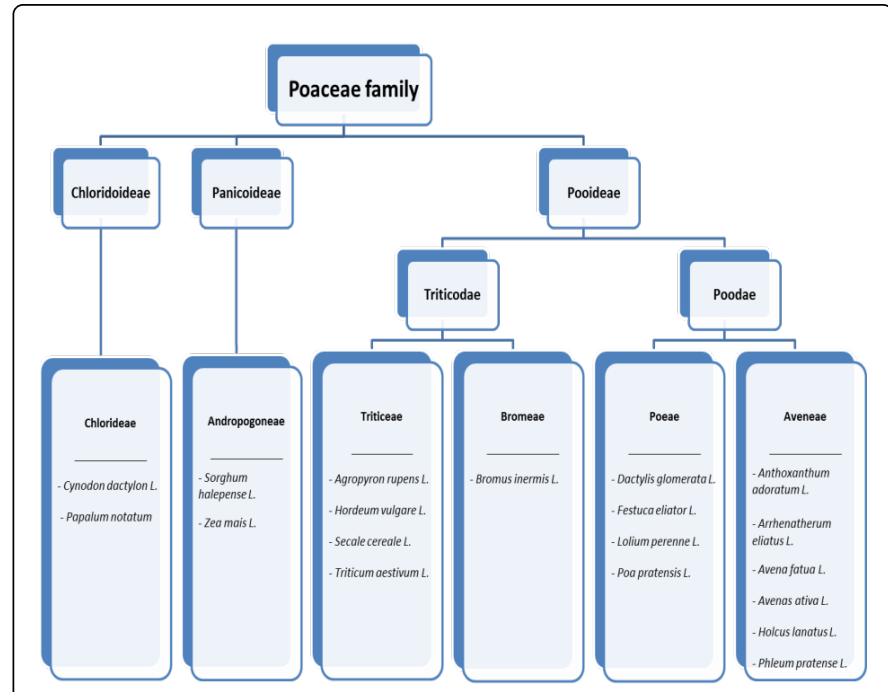

Figure 1: Taxonomy of the Poaceae family of grasses.

\section{Case Report}

Here we report the case of a patient who after three years of AIT developed a negative response to allergy tests to the specific allergens administered with the treatment. The patient was a 39 years old woman suffering from four years of persistent rhinitis and intermittent asthma caused by sensitization to grass pollen. The skin prick test (SPT) (Stallergenes, Antony, France) was positive for grass pollens, with a $3+$ response to Phleum pratense, Dactylis glomerata and Cynodon dactylon. The values of specific IgE to such pollens, measured by CAP System (Thermo Fisher Scientific, Uppsala, Sweden) were $37.5 \mathrm{kU} / \mathrm{L}$ for $P$. pratense, $42.1 \mathrm{kU} / \mathrm{L}$ for $D$. glomerata and 32.6 $\mathrm{kU} / \mathrm{L}$ for $C$. dactylon. The patient was treated with SCIT using a grass pollen extract (Phostal, Stallergenes, Antony, France) containing $P$. pratense, D. glomerata, Anthoxanthum odoratum, Poa pratensis and Lolium perenne but not $C$. dactylon. After SCIT initiation, the patient showed a significant improvement of allergic symptoms since the first year. In particular, in the first grass pollen season following the start of SCIT he had no more asthmatic symptoms, while nasal symptoms were progressively reduced during SCIT. No adverse reactions occurred during the treatment. After three years, the patients was free of both nose and lung symptoms during the grass pollen season. The absence of symptoms during the grass pollen season persisted over time. However, the patients referred for a new visit for allergy because but in the latest year she had rhinitis from mid-August to early October. SPT were performed, with a negative results for $P$. pratense and $D$. glomerata and positive (2+) for $C$. dactylon. Specific IgE values were $<0.10 \mathrm{kU} / \mathrm{L}$ for $P$. pratense and $D$. glomerata and $24.7 \mathrm{kU} / \mathrm{L}$ for $C$. dactylon (Figure 2). A positive result to SPT (3+) was found instead for ragweed pollen, thus a new immunotherapy with a ragweed extract was planned.

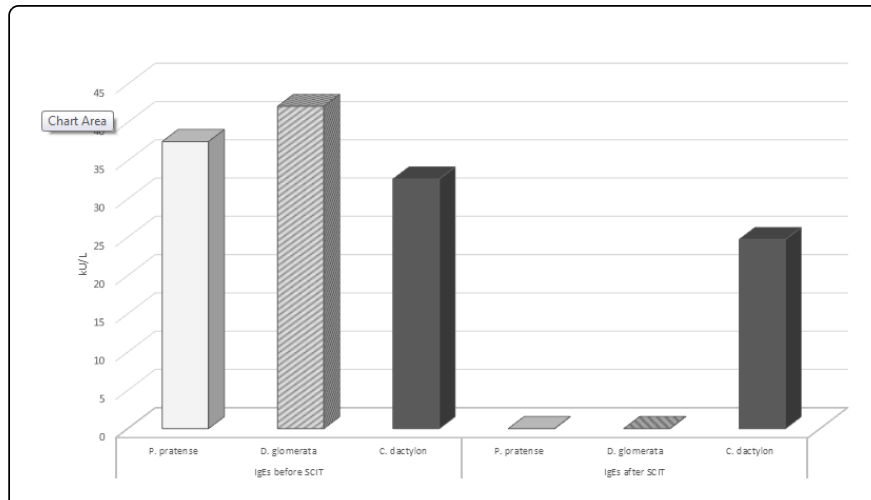

Figure 2: The measurement of specific IgE before and after SCIT shows the achievement of completely negative tests to the grasses contained in the extract used for immunotherapy.

\section{Discussion}

Specific allergen immunotherapy (AIT) is the only diseasemodifying treatment for AR, based on its ability to alter the Th2influenced immune response, while pharmacotherapy acts only on symptoms $[17,18]$. The capacity of AIT to improve the symptoms of respiratory allergy and to decrease the consumption of drugs is clearly demonstrated by meta-analyses that included a large number of randomised, double-blind, placebo controlled trials [19]. Such outcome is founded on the modification of the immunological response to the administered allergen. In the early 1990s, the definition of the Th1-Th2 paradigm paved the way to a deeper understanding of mechanisms of action of AIT, as well described in complete reviews $[20,21]$. The first observations, obtained by both in vitro and in vivo models concluded that SCIT turned the allergen-specific responses from the Th2 allergic phenotype to a tolerogenic Th1 phenotype. Based on the fact that alterations in circulating $\mathrm{T}$ cells do not mirror local response in target organs, a redirection induced by SCIT in respiratory mucosa was suggested. In fact, in SCIT-treated patients the T cells expressing IFN- $\gamma$ mRNA were increased in the nasal mucosa after pollen challenge. Also in nasal biopsies and in nasal fluid of SCITtreated patients the increase of IFN- $\gamma$ and the concomitant decrease of IL-5 and IL-9 during the pollen season was apparent. The discovery of regulatory T cells (Treg) further expanded the knowledge, highlighting that their increased activity (immunosuppression) was the main or mechanism explaining the clinical efficacy of SCIT. The suppressor activity of Treg is mediated by regulatory cytokines including IL-10, TGF- $\beta$ and IL-35. In particular, during pollen exposure CD4+CD25+ Treg cells from grass-sensitive patients were shown to be weakened in suppressing IL-13 and IL-5 production compared to healthy controls. These AIT-induced changes in immunological response to allergens have an important outcome in the prolonged effect of immunotherapy over time once the treatment is discontinued [22].

The drawback of SCIT has always been the risk of systemic reactions that rarely may also be life-threatening [23]. However, recent studies showed that, if SCIT is performed following strict safety rules, the risk of systemic reactions is very low [24]. Indeed, the new option of administering the allergen extracts by the sublingual route was mainly introduced in the 1980s for safety reasons [11]. The good safety profile of SLIT, the common side effects being local reactions in the mouth, while systemic reactions are very rare [25], allows the administration 
of the treatment at home and makes SLIT less time-consuming than SCIT. Notwithstanding, when patients receive complete information on the characteristic of SCIT and SLIT before starting the treatment, the rate of patients who choose SCIT is comparable to that of patients choosing SLIT [26].

The findings from this case show that SCIT in optimal circumstances is able to achieve a complete tolerance to the administered allergen demonstrated by the development of negative results to the grass pollens contained in the extract used for the treatment. Instead, specific IgE to the pollen not included in the extract, i.e. C. dactylon, showed a decrease in respect to basal value, that is likely to be related to the natural history of allergy, while maintaining the positive response to SPT.

Natural history is claimed to be able to achieve similar results. In 2003, studies addressing the effects of aging on both sensitization to Japanese cedar pollen (JCP) and development of JC pollinosis, as assessed by serum IgE titers to JCP and clinical examination were conducted in Japan. In a vertical-sectional study, the serum IgE titer to JCP was significantly influenced by the amounts of pollen in the air. An increase in age by 6 years did not reduce serum IgE to JCP in subjects aged up to 40 years, while in subjects with JC pollinosis aged over 40 with a moderate-high IgE titer, spontaneous remission of JC pollinosis was observed in $16.1 \%$ over a period of 6 years [27]. However, the literature on spontaneous remission of pollen allergy is quite scarce. In particular, no study suggesting a selective development of negative allergy tests for some pollens but not for others are available, while thus occurred in our patient due to the lack in the extract of the pollen to which the patient was also sensitized.

\section{Conclusion}

This case report offers data confirming the recent definition of AIT as a treatment fulfilling the requirements of precision medicine [28], which is an innovative approach to medical diagnosis and treatment that is likely to be extensively applied in the next years [29].

\section{Conflicts of Interest}

Conflict of interest is a scientific consultant for Stallergenes Italy. E.B., E.M. D.P. and M.M. declare that they have no competing interests related to the content.

\section{References}

1. D’Amato G, Cecchi L, Bonini S, Nunes C, Maesano AI, et al. (2007) Allergenic pollen and pollen allergy in Europe. Allergy 62: 976-990.

2. Rodríguez-Rajo FJ, Astray G, Ferreiro-Lage JA, Aira MJ, Jato-Rodriguez MV, et al. (2010) Evaluation of atmospheric Poaceae pollen concentration using a neural network applied to a coastal Atlantic climate region. Neural Netw 23: 419-425.

3. Quercia O, Incorvaia C, Puccinelli P, Scurati S, Emiliani F, et al. (2012) Prevalence of allergic disorders in Italy: the Cotignola population study. Eur Ann Allergy Clin Immunol 44: 5-11.

4. Davies JM, Beggs PJ, Medek DE, Newnham RM, Erbas B, et al. (2015) Trans-discliplinary research in synthesis of grass pollen aerobiology and its importance for respiratory health in Australasia. Sci Total Environ 534: 85-96.

5. Genuneit J, Seibold AM, Apfelbacher CJ, Konstantinou GN, Koplin JJ, et al. (2017) Task Force "Overview of Systematic Reviews in Allergy Epidemiology (OSRAE)" of the EAACI Interest Group on Epidemiology. Overview of Systematic Reviews in Allergy Epidemiology. Allergy 72: 849-856.
6. Salcedo G, Quirce S, Diaz-Perales A (2011) Wheat allergens associated with Baker's asthma. J Investig Allergol Clin Immunol 21: 81-92.

7. Cecchi L, Dell'Albani I, Frati F (2013) Towards a global vision of molecular allergology: a map of exposure to airborne molecular allergens. Eur Ann Allergy Clin Immunol. 45: 17-23.

8. Frenguelli G, Passalacqua G, Bonini S, Fiocchi A, Incorvaia C, et al. (2010) Bridging allergologic and botanical knowledge in seasonal allergy: a role for fenology. Ann Allergy Asthma Immunol 105: 223-227.

9. Popescu FD (2014) Molecular biomarkers for grass pollen immunotherapy. World J Methodol 4: 26-45.

10. San Nicolò M, Braun T, Eder K, Bergaus A, Groger M, et al. (2016) Clinical relevance of $\operatorname{IgE}$ to profilin and polcalcin in polle-sensitized patients. Int Arch Allergy Immunol 169: 101-107.

11. Incorvaia C, Frati F (2011) One century of allergen-specific immunotherapy for respiratory allergy. Immunotherapy 3: 629-635.

12. Matricardi PM, Kuna P, Panetta V, Wahn U, Narkus A, et al. (2011) Subcutaneous immunotherapy and pharmacotherapy in pollen-induced seasonal allergic rhinoconjunctivitis. J Allergy Clin Immunol 128: 791-799.

13. Devillier P, Dreyfus JF, Demoly P, Calderón MA (2014) A meta-analysis of sublingual allergen immunotherapy and pharmacotherapy in polleninduced seasonal allergic rhinoconjunctivitis. BMC Med 12: 71.

14. Nelson H, Cartier S, Allen-Ramey F, Lawton S, Calderon MA, et al. (2015) Network meta-analysis shows commercialized subcutaneous and sublingual grass products have comparable efficacy. J Allergy Clin Immunol Pract 3: 256-266.

15. Würtzen PA, Gupta S, Brand S, Andersen PS (2016) Grass pollen immunotherapy: where are we now. Immunotherapy 8: 399-411.

16. Rawle FC, Burr ML, Platts-Mills TA (1983) Long-term falls in antibodies to dust mite and pollen allergens in patients with asthma or hay fever. Clin Allergy 13: 409-417.

17. Canonica GW, Passalacqua G (2011) Disease-modifying effect and economic evaluation of sublingual immunotherapy. J Allergy Clin Immunol 127: 44-45.

18. Viswanathan RK, Busse WW (2012) Allergen immunotherapy in allergic respiratory diseases: from mechanisms of action to meta-analyses. Chest 141: 1303-1314.

19. Compalati E, Penagos M, Tarantini F (2009) Specific immunotherapy for respiratory allergy: state of the art according to current meta-analyses. Ann Allergy Asthma Immunol 102: 22-28.

20. Maggi E (2010) T-cell responses induced by allergen-specific immunotherapy. Clin Exp Immunol 161: 10-18.

21. Jutel M, Akdis CA (2011) Immunological mechanisms of allergen-specific immunotherapy. Allergy 66: 725-732.

22. Frati F, Dell'Albani I, Incorvaia C (2013) Long-term efficacy of allergen immunotherapy: what do we expect?. Immunotherapy 5: 131-133.

23. Amin H, Liss G, Bernstein D (2006) Evaluation of near-fatal reactions to allergen immunotherapy injections. J Allergy Clin Immunol 117: 169-175.

24. Schiappoli M, Ridolo E, Senna G (2009) A prospective Italian survey on the safety of subcutaneous immunotherapy for respiratory allergy. Clin Exp Allergy 39: 1569-1574.

25. Gidaro GB, Marcucci F, Sensi L, Incorvaia C, Frati F, et al. (2005) The safety of sublingual swallow immunotherapy: an analysis of published studies. Clin Exp Allergy 35: 565-571.

26. Manzotti G, Pappacoda A, Dimatteo M, Scolari C, Riario-Sforza GG, et al. (2013) Ultra short pre-seasonal subcutaneous and pre-coseasonal sublingual immunotherapy for pollen allergy: an evaluation of patient's preference in real life. Eur Ann Allergy Clin Immunol 45: 138-144.

27. Okawa T, Konno A, Yamakoshi T, Numata T, Terada N, et al. (2003) Analysis of natural history of Japanese cedar pollinosis. Int Arch Allergy Immunol 131: 39-45.

28. Canonica GW, Bachert C, Hellings P, Ryan D, Valovirta E, et al. (2015) Allergen Immunotherapy (AIT): a prototype of precision medicine. World Allergy Organ J 8: 31 . 
Citation: Boni E, Incorvaia C, Makrì E, Preziosi D, Mauro M (2017) When Allergen Immunotherapy Perfectly Meets its Need: A Case Report. J Allergy Ther 8: 259. doi:10.4172/2155-6121.1000259

Page 4 of 4

29. Muraro A, Lemanske RF Jr, Hellings PW, Akdis CA, Bieber T, et al. (2016) Precision medicine in patients with allergic diseases: airway diseases and atopic dermatitis - PRACTALL document of the European Academy of
Allergy and Clinical Immunology and the American Academy of Allergy, Asthma and Immunology. J Allergy Clin Immunol 137: 1347-1358. 